\title{
Implementación y adaptación en Colombia del sistema preventivo Communities That Care
}

\section{Implementation and adaptation in Colombia of the Communities That Care}

\author{
Juliana Mejía-Trujillo*, Augusto Pérez-Gómez**, María Fernanda Reyes-Rodríguez*** \\ *Trabajadora Social de la Corporación Nuevos Rumbos. **Ph.D., Universidad Católica de Lovaina, Director de la Corporación \\ Nuevos Rumbos. *** MsC, Universidad de Liverpool, Psicóloga Corporación Nuevos Rumbos.
}

\section{Resumen}

La Corporación Nuevos Rumbos (Colombia) viene implementando hace más de dos años en ocho comunidades de Colombia, el sistema preventivo Comunidades Que se Cuidan (CQC), adaptación de Communities That Care (CTC), creado en la Universidad de Washington en Seattle, que ha sido desarrollado por más de 25 años en los Estados Unidos y en ocho países de América, Oceanía y Europa. El sistema busca que, a través del empoderamiento comunitario y empleando el enfoque de la Salud Pública y en la estrategia de desarrollo social, las comunidades tomen las mejores decisiones basadas en los datos de prevalencias de consumo y en la identificación de los factores protectores y de riesgo (basada en la utilización de la encuesta original validada en Colombia) y puedan escoger las estrategias de intervención probadas que más se ajusten a sus necesidades. Este documento describe el proceso de implementación en Colombia, sus diferencias con CTC, la creación de puntos de corte propios para el país, las principales limitaciones en el proceso de adaptación y cómo se abordaron. CQC aparece como un sistema preventivo que puede tener amplia aplicabilidad en otros países de América Latina.

Palabas clave: Comunidades Que se Cuidan, prevención, consumo de drogas, factores de riesgo, factores protectores.

\begin{abstract}
For more than two years, Corporación Nuevos Rumbos (Colombia) has been carrying out, in eight Colombian communities, a preventive system called Comunidades Que se Cuidan (CQC), an adaptation of Communities That Care (CTC), created at the University of Washington (Seattle), developed for more than 25 years in the United States of America and implemented in eight countries of America, Oceania, and Europe. The system is based on the public health approach, and the social development strategy for community empowerment. The core idea is to teach communities how to make decisions based on data regarding drugs and alcohol consumption and the identification of protective and risk factors, on the basis of the original survey validated in Colombia: these will allow communities to choose the best preventive interventions, tailored for each of them according to their needs. This paper describes the process of implementation of CQC in Colombia, its differences with CTC, the creation of Colombian cutpoints, the main difficulties and how these were solved. CQC seems to be a preventive system with a wide potential applicability in other Latin American countries.

Key Words: Communities That Care (CTC), prevention, drug use, risk factor, protective factors
\end{abstract}




\section{Surgimiento de Communities That Care (CTC)}

A finales de la década de los 80, J. David Hawkins y Richard Catalano, profesores de la Universidad de Washington en Seattle, crearon un sistema reventivo denominado Communities That Care (CTC). Este sistema, basado en la Estrategia de Desarrollo Social y en el Enfoque de Salud Pública, se soporta también en la integración de tres grandes elementos: El trabajo articulado hacia el empoderamiento comunitario, la aplicación regular de la "Encuesta para Jóvenes", y un "Menú" de programas probados y efectivos, disponibles para las comunidades (Catalano, Arthur, Hawkins, Berglund y Olson, 1998; Hawkins, Catalano y Arthur, 2002).

CTC cuenta con cerca de 25 años de implementación y evaluación permanentes, soportando conceptual y teóricamente su desarrollo con la recolección de datos empíricos de alta calidad, lo que le ha generado el reconocimiento de ser uno de los mejores sistemas preventivos del mundo occidental (Brown, Hawkins, Arthur, Briney y Abbot, 2007; Brown, Hawkins, Arthur, Briney y Fagan, 2011; Feinberg, Greenberg, Osgood, Sartorius y Bontempo, 2007; Hawkins, Catalano y Miller, 1992; Rhew, Brown, Hawkins, y Briney, 2013; Social Development Research Group, 2014). Así mismo, CTC está siendo implementado en Estados Unidos (más de 400 comunidades), Canadá, Australia, Países Bajos, Alemania, Reino Unido, Croacia y Chipre. En España se ha utilizado uno de sus principales instrumentos, la encuesta, para medir prevalencias de consumo de drogas y factores protectores y de riesgo (López y Rodríguez, 2010) y en Chile y en Brasil se está validando. India, Suecia y países de Centro América (Guatemala, Honduras y El Salvador) han mostrado interés o están utilizando algunos elementos del sistema.

\section{CTC como sistema preventivo}

Este sistema se basa en el concepto de Coaliciones comunitarias, que permiten la reducción de la desorganización social; la promoción de normas comunitarias efectivas contra el consumo de sustancias psicoactivas, la delincuencia y otros comportamientos problemáticos de los adolescentes (violencia, fracaso escolar y embarazo); y la apropiación por parte de la comunidad de las actividades preventivas (France y Crow, 2005). Por tener como eje principal a la comunidad, un buen número de objetivos están centrados en la organización de ésta y en la capacitación a los miembros de las Juntas Comunitarias; otros objetivos se relacionan con la implementación de programas efectivos y la evaluación permanente de los factores protectores y de riesgo y de los comportamientos problemáticos. Los principales objetivos son los siguientes:

- Proveer capacitación a las comunidades en conceptos relacionados con la ciencia de la prevención y el ejercicio de planeación basada en los datos;

- Identificar, priorizar, evaluar y monitorear factores protectores y de riesgo en cada comunidad, a través de la aplicación de un instrumento y de la utilización de datos de archivo locales, para una planeación focalizada;

- Crear redes interinstitucionales y un lenguaje común de prevención;

- Implementar programas probados y efectivos enfocados en los factores protectores y de riesgo prioritarios en las comunidades, para disminuir comportamientos problemáticos en los adolescentes;

- Evaluar los recursos existentes en la comunidad y las intervenciones realizadas a partir del plan de acción.

La razón por la cual CTC es un sistema y no un programa, hace referencia a la articulación de los procesos de empoderamiento comunitario, el desarrollo de perfiles comunitarios y la construcción de un menú de programas probados y efectivos. Estos elementos se explican a continuación:

- Un proceso de empoderamiento comunitario en el que, a lo largo de las cuatro primeras fases, se consolidan entre sus miembros las nociones relacionadas con la ciencia de la prevención, y también las relaciones individuales e institucionales necesarias para soportar la implementación y el monitoreo de un plan de acción;

- La elaboración de un perfil comunitario basado en datos de archivo de las comunidades y en un instrumento de diagnóstico denominado "Encuesta para jóvenes", que fue probado en siete estados de los Estados Unidos, con una muestra suficiente de estudiantes ( $\mathrm{n}=172.628$; Briney, Brown, Hawkins y Arthur, 2012; Brown, Graham, Hawkins, Arthur, Baldwin, Oesterle, Briney, Catalano y Abott, 2009; Glaser, van Horn, Hawkins, Arthur y Catalano, 2005). El cuestionario original de CTC está dirigido a evaluar 25 factores de riesgo (FR) y 13 factores protectores (FP) en los cuatro dominios propuestos por CTC (familia, comunidad, escuela y pares e individuo: Arthur, Hawkins, Brown, Briney, Oesterle y Abbott, 2010; Arthur, Hawkins, Pollard, Catalano y Baglioni, 2002; Hawkins, 2006) y ubica a los estudiantes en alto y bajo riesgo o protección a través de puntos de corte. En Estados Unidos las características psicométricas del cuestionario han sido ampliamente estudiadas; los resultados han mostrado que este instrumento cuenta con una alta confiabilidad, así como una buena validez de constructo y predictiva tanto para hombres como para mujeres, así como en diferentes grupos étnicos incluyendo latinos (Arthur et al, 2002; Briney, Brown, Hawkins y Arthur, 2012; Glaser, Van Horn, Arthur, Hawkins y Catalano, 2005). En el caso colombiano, la encuesta para jóvenes evalúa, a través de 103 reactivos, los comportamientos de consumo de SPA y el perfil de riesgo y protección, divididos en 11 de los 23 factores de riesgo de la encuesta original, y tres factores protectores (Ver Tabla 1);

- La construcción de un “menú” de programas preventivos que han sido sometidos a evaluaciones y que cada Junta Comunitaria tiene como base para hacerle frente a los factores de riesgo prioritarios, resultado de la elaboración del perfil de la comunidad. 


\section{Objetivo}

Describir el proceso de implementación y adaptación de CTC a las condiciones socioculturales de Colombia, señalando por qué no es posible utilizar el sistema de manera idéntica a como se utiliza en el país donde fue creado.

\section{Fases de CTC}

El sistema se encuentra dividido en cinco fases cíclicas:

1. Inicio: se evalúa la disponibilidad para el cambio en la comunidad, se identifican actores, se reclutan líderes y se obtiene el apoyo de las escuelas para aplicar la encuesta sobre factores protectores y de riesgo.

2. Organizar, introducir, involucrar: se forma una coalición y se da inicio a las capacitaciones de los actores adultos principales, que se prolongarán durante las cinco fases; se desarrolla una visión de futuro para los niños de la comunidad y se organiza una estructura para poder moverse en esa dirección. Se hacen dos capacitaciones: la orientación a líderes sobre prevención y la forma de organizar un Comité Comunitario que sea la máxima autoridad a la hora de tomar decisiones.

3. Desarrollo de un perfil de la comunidad: fase en la que se aplica la encuesta a jóvenes; los líderes escogidos reciben una capacitación sobre la forma de interpretar los factores protectores y de riesgo y sobre esa base sugieren cuáles son prioritarios. También se evalúan los programas comunitarios, las políticas y los recursos existentes referentes a los factores protectores y de riesgo.

4. Plan de acción: La Junta Comunitaria revisa los resultados de la fase 3 y desarrolla un plan de acción, a partir de un entrenamiento en planeación comunitaria. Se escogen los programas, las prácticas y las políticas que puedan cambiar los factores de riesgo y los comportamientos problemáticos. Se proponen metas y objetivos medibles.

5.Implementación: se ofrece un entrenamiento para la ejecución del plan comunitario considerando la importancia de mantenerse fiel a los lineamientos del sistema, de los aspectos financieros de la implementación, y de los elementos que permitirán evaluar adecuadamente los resultados.

\section{La implementación de CTC en Colombia 2011-2014}

Colombia es un país en el que el problema del consumo de drogas ha tenido un impacto importante, que se ha visto incrementado por fenómenos como el narcotráfico y la guerrilla (Pérez Gómez, 2013). Igualmente, tal como lo han demostrado la OPS (Monteiro, 2013), y los estudios nacionales (Ministerio de Justicia y del Derecho., Ministerio de Educación Nacional, y Ministerio de Salud y Protección Social, 2011), el país tiene un elevado consumo de alcohol, que afecta particularmente a poblaciones muy jóvenes.

A pesar de que en el país no ha habido grandes desarrollos en el ámbito de la prevención, los cambios significativos en esta área que le dan gran importancia a las dimensiones comunitaria y neuropsicológica (Sloboda, 2014), han atraído la atención de las autoridades nacionales y de las entidades que se ocupan de la prevención.

En el año 2007 Nuevos Rumbos estableció contacto con el Social Development Research Group (SDRG) de la Universidad de Washington, con el fin de conocer más sobre el sistema preventivo y determinar las condiciones para llevarlo a cabo en Colombia; este contacto llevó a crear una versión en español de la encuesta para jóvenes y a la traducción de todo el material disponible de CTC por parte del equipo de Nuevos Rumbos, con el soporte permanente de los creadores del sistema. Los esfuerzos se vieron recompensados en el año 2011, cuando la Organización Panamericana de la Salud (OPS) y el Ministerio de Salud y Protección Social (MSPS), invitaron a la Corporación a llevar a cabo el pilotaje del sistema en dos comunidades del país, bajo el nombre "Comunidades Que se Cuidan" - CQC -. Una de las comunidades estaba compuesta por un grupo de barrios de la ciudad de Bogotá y otra era un municipio a $25 \mathrm{~km}$ de distancia. Ocho meses después, una tercera comunidad del departamento de Cundinamarca fue añadida a la experiencia de pilotaje, y en esta oportunidad el sistema se insertó en el comité de sustancias psicoactivas del municipio. Año y medio después de la primera experiencia, con el apoyo del Instituto Colombiano de Bienestar Familiar, se inició la implementación de las cuatro primeras fases de CQC en cinco municipios de la zona cafetera, en donde se evidencian altos niveles de consumo de alcohol y drogas entre los adolescentes, así como otros problemas sociales.

En general se intentó mantener una alta fidelidad a CTC, pero dadas las diferencias socioculturales de las comunidades en comparación con Estados Unidos, se introdujeron algunos cambios que se describen a continuación:

1. En fase uno, para asegurar la adecuada implementación del sistema, Nuevos Rumbos decidió coordinar, capacitar y acompañar personalmente a las comunidades, mientras que en CTC el entrenador es contratado por una entidad, por lo general una ONG, que cumple a cabalidad las funciones de coordinador y responsable del proceso en esa comunidad (Brooke-Weiss, Haggerty, Fagan, Hawkins, y Cady, 2008). Si bien las Administraciones Municipales, como aporte local, designaron a algunos de sus funcionarios como coordinadores, sus funciones estaban muy lejos de ser las esperadas por el sistema, pues Nuevos Rumbos se encargaba de la convocatoria, las reuniones con los líderes clave, las reuniones de la Junta Comunitaria, la definición de estrategias para atraer nuevos miembros al grupo, entre otras. Cuando el primer ciclo del sistema fue implementado, los coordinadores comenzaron a asumir funciones mucho más comprometidas y responsables, esta vez con el acompañamiento, mas no con el liderazgo, de Nuevos Rumbos. 
2. Otro elemento importante en el desarrollo de la fase uno, fue la construcción de un instrumento ágil y efectivo de medición de disponibilidad de la comunidad, pues en los encuentros iniciales siempre se expresa mucho interés por participar en la Junta Comunitaria; sin embargo, puede suceder que quienes allí se encuentran no representen todos los sectores de la comunidad, no sean verdaderos líderes que sostengan el proceso a futuro o el momento por el que pasa la comunidad no sea el óptimo para dar inicio a CQC en su municipio. Este instrumento principalmente mide la disponibilidad de los líderes, de la comunidad en general y la fortaleza de los lazos comunitarios, y está basado en un instrumento más sencillo elaborado por la Universidad de Washington (Arthur, Hawkins, Catalano y Olson, 2002)

La segunda parte de la fase dos (Orientación a la Junta Comunitaria - OJC -), se realizó pocas semanas después de presentar el sistema a los líderes clave, mientras que en CTC se hace entre uno y tres meses después; el objetivo es que los líderes tengan el tiempo, junto con el coordinador contratado y la agencia contratante, de invitar y jalonar el proceso de convocatoria de quienes serán los miembros de la Junta Comunitaria (BrookeWeiss et al, 2008; SDRG, 2014).

Adicionalmente, CQC durante su pilotaje, no dividió el grupo general en subgrupos de trabajo, pues la asistencia permanente a las reuniones era de un número reducido de personas $(12-15)$, insuficientes para el desarrollo de los seis grupos de trabajo propuestos por CTC (datos, financiación, evaluación, relaciones públicas, mantenimiento de la coalición e involucramiento juvenil: SDRG, 2014). Una vez finalizada la fase 4, debido al conocimiento aún más cercano de la experiencia y al contacto con Maestros de Entrenadores de los Estados Unidos, tres de las ocho juntas comunitarias constituidas a la fecha conformaron subgrupos de trabajo, con el fin de asegurar la continuación del sistema una vez Nuevos Rumbos se vaya de las comunidades; los grupos de trabajo fueron Mantenimiento, Financiación y Evaluación. El primer grupo se preocupa por atraer nuevos miembros y capacitar en los aspectos básicos de CQC, el segundo, de conseguir financiación permanente para la implementación de los planes de acción, y el tercero se encarga de diseñar y ejecutar los planes de evaluación de los programas y prácticas seleccionadas en la fase $4 \mathrm{y}$ del sistema en general.

3. La construcción de perfil de la comunidad en la fase 3, comenzó desde el momento en el que se estableció contacto con las autoridades locales, de modo que se contara con los datos cuando las comunidades, entre 2 y 3 meses después de iniciado el proceso, estuvieran en la elaboración del mapa de la comunidad, en cuanto a consumo de SPA y a factores protectores y de riesgo en los cuatro ámbitos de prevención. La capacitación de esta fase se llevó a cabo con el grupo en pleno de la Junta Comunitaria y no por subgrupos como se tiene previsto en CTC, lo que, en la mayoría de los casos, generó un retraso en el desarrollo pues no todos los miembros estaban en la capacidad o tenían el interés de apropiarse de la recolección y análisis de datos de manera permanente. La división por subgrupos, aseguraría que estas labores quedaran en manos de epidemiólogos o encargados del seguimiento de los fenómenos sociales de cada comunidad, y tuvieran un manejo y un uso adecuados.

4. Para llevar a cabo el proceso de planeación en la fase cuatro, debe elaborarse un menú de programas (Fagan, van Horn, Hawkins y Arthur, 2007; Ringwalt, Vincus, Hanley, Ennett, Bowling y Haws, 2010). La construcción del nuestro no incluyó evaluaciones de diseño experimental, ya que ni en Colombia ni en América Latina son comunes este tipo de evaluaciones; por el contrario, se cuenta con evaluaciones de diseño cuasi-experimental y con éstas se hizo el menú de programas que las comunidades utilizan para la selección de programas a implementar en la fase cinco; el resultado fue el siguiente: hay 15 programas utilizados en el país, pero solo uno (Anímate) cuenta con evaluación sistemática durante 13 años con nueve mediciones (Aja Eslava y Gómez Avila, 2013); siete programas tienen alguna clase de monitoreo (percepción de riesgo, información sobre herramientas de evaluación, sistematizaciones o líneas de base y de cierre; tales programas son: Consentidos, Sanamente, Familias Fuertes, Leones Educando, Habilidades para la Vida, CEMA-PEMA/ NEF-PIP, Zonas de Orientación Escolar), y siete no cuentan con evaluación (Colombia Joven, Experiencias para Vivir y Convivir, ACJ-YMCA, Fundación CreSer, Caminos, Retomemos y DARE) ${ }^{1}$. Al momento de formular el plan de acción, las comunidades escogieron implementar los dos programas más adelantados en su evaluación (Anímate y Consentidos). Para subsanar esta dificultad, las Juntas también identificaron otras prácticas que pudieran responder a los factores prioritarios identificados en la fase 3 , y se contemplaron algunas estrategias de cambio de metodología para facilitar la implementación de los programas y las prácticas seleccionadas. Así mismo, Nuevos Rumbos ofreció un conjunto de estrategias para apoyar las que ya estaban disponibles en la comunidad:

- Sesiones de capacitación para líderes comunitarios, padres de familia y maestros, con el fin de clarificar

1 A finales de 2014 Nuevos Rumbos recibió capacitación en un programa de probada efectividad con población hispana en los Estados Unidos, "Familias que se cuidan: encaminándolos hacia buenas decisiones". Hay contactos con la GIZ de Alemania, cuyo programa Miles de Manos está siendo utilizado en Centro América. 
y unificar conceptos relacionados con prevención; en ellas se explican los factores protectores y de riesgo, la importancia de las coaliciones para la solución de problemas sociales y la importancia de abordar el consumo de alcohol y drogas en los adolescentes, desde la perspectiva de salud pública.

- Estrategias para el uso adecuado del tiempo libre: La comunidad desarrolla opciones para que los adolescentes ocupen su tiempo de manera productiva, en los momentos en que son más propensos al consumo de alcohol y otras SPA; entre ellas se contemplan actividades deportivas y culturales que las comunidades crean de acuerdo con los intereses de sus habitantes.

- Intervenciones breves:

- IBEM (Intervención Breve basada en Entrevista Motivacional): se implementa con adolescentes escolarizados, en su mayoría de grados octavo y noveno. El programa consta de una primera intervención, dos seguimientos y dos intervenciones con padres, inspirados en la entrevista motivacional, y acompañados de un instrumento de tamizaje (CRAFFT/CARLOS) (Harris, Csémy, Sherritt, Starostova, Van Hook, Johnson, Boulter, Brooks, Carey, Kossack, Kuligm, Vraken y Knight, 2012; Knight, Sherritt, Harrys, Gates y Chang, 2003; Pérez-Gómez y Scoppetta, 2011; Substance Abuse and Mental Health Services Administration, 1999). Este último instrumento ubica al estudiante en un nivel de riesgo que sirve para que cada uno de los participantes se plantee una meta de disminución de consumo o retraso en su edad de inicio. Los seguimientos se desarrollan con base en esta meta autoimpuesta y las intervenciones con padres se desarrollan con base en los factores de riesgo que se encontraron más predominantes en cada colegio.

- "Cápsulas de humor": Son videos de corta duración (3 minutos), en los que un actor conocido ridiculiza situaciones relacionadas con el consumo de alcohol o de otras sustancias. Los videos abren la reflexión a partir de la identificación con el personaje y sirven además para desmitificar asuntos relacionados con el consumo y clarificar dudas.

- Profesión papás: Se trata de un libro preparado para padres de familia, con información sobre alcohol y drogas, sexualidad, trastornos alimenticios, matoneo ('bullying') y otros problemas propios de la adolescencia, así como tips de manejo de situaciones específicas y pequeños cuestionarios que ayudan a los padres a identificar y actuar frente a estas situaciones.

- Entrenamiento básico a la comunidad en procesos de evaluación, con el fin de que ellos mismos lleven a cabo o monitoreen la evaluación de sus programas y prácticas, como uno de los pilares de CQC.

- Utilización de la práctica 'Mystery Shopper' (“Comprador Aliado”), con el fin de evitar la venta de alcohol a menores de edad y como una forma de control social.

En las guías de CTC, cada una de las fases tiene una duración de uno a tres días, con una intensidad aproximada de ocho horas diarias; en CQC las capacitaciones se desarrollan según los acuerdos específicos a los que se llegue en cada comunidad, y por lo general se programan semanal o quincenalmente, con una duración de dos y cuatro horas cada encuentro. Esto se plantea porque un buen número de integrantes de las Juntas Comunitarias no son voluntarios, y deben cumplir con funciones públicas que no les permiten disponer de varios días cada mes, para asistir a las capacitaciones.

A lo largo de la implementación de CQC en ocho comunidades, es posible determinar que tienen más éxito aquellas cuya fase cinco puede ser financiada en forma inmediata, con recursos propios o externos. Lo ideal es que las comunidades, durante las cuatro primeras fases de CQC, reestructuren y optimicen el presupuesto destinado a prevención, pero esta vez focalizado a los factores prioritarios, identificados en el instrumento para la construcción del perfil de la comunidad.

La falta de datos de archivo en los pequeños municipios de Colombia, potenciales comunidades para implementar CQC, es una de las mayores dificultades; por ello las cifras de delincuencia juvenil, consumo de drogas o incidentes asociados a consumo de alcohol en esos municipios, se han reemplazado con información no oficial o con indicadores indirectos.

\section{Instrumento}

Actualmente el cuestionario de CQC (disponible en www. nuevosrumbos.org) cuenta con un análisis de confiabilidad y validez de constructo, el cual se realizó con los datos de 33.790 estudiantes. Los resultados mostraron una alta y moderada consistencia interna en los FR y alta en los tres FP (ver Tabla 1). Actualmente, está en proceso el análisis de la validez de constructo a través de un análisis factorial confirmatorio para datos ordinales.. Este proceso se realiza bajo el marco de un proyecto con la Universidad de Washington y NIDA, los resultados serán revelados en futuras publicaciones. Hasta el momento, el ajuste de los modelos de medición de cada factor de riesgo y protección han mostrado un ajuste adecuado con resultados menores o iguales a 0.08 para Root Mean Square Error of Approximation (RMSEA) e iguales o mayores a 0.95 para Comparative Fit Index (CFI) y Tucker Lewis Index (TLI). 
Tabla 1

Coeficientes de confiabilidad de los factores de riesgo y de protección evaluados en la encuesta para jóvenes de CQC

\begin{tabular}{lc}
\hline Factores de riesgo & $\begin{array}{c}\text { Alfa de } \\
\text { Cronbach }\end{array}$ \\
\hline Percepción sobre la Disponibilidad de Drogas & 0.81 \\
Reglas y Normas Favorables al Uso de Drogas & 0.69 \\
Actitudes de los padres hacia el Uso de Drogas & 0.57 \\
Pobre Manejo Familiar & 0.83 \\
Bajo compromiso al colegio & 0.70 \\
$\begin{array}{l}\text { Percepción de Riesgo del uso de Drogas de los jóvenes } \\
\text { Actitudes favorables de los jóvenes Hacia el Uso de }\end{array}$ & 0.70 \\
$\begin{array}{l}\text { Drogas } \\
\text { Comportamiento Antisocial }\end{array}$ & 0.79 \\
$\begin{array}{l}\text { Uso de Drogas en Amigos } \\
\text { Comportamientos Antisociales en Amigos }\end{array}$ & 0.86 \\
\hline $\begin{array}{l}\text { Actitudes favorables de los padres hacia el } \\
\text { comportamiento antisocial }\end{array}$ & 0.76 \\
\hline $\begin{array}{l}\text { Factores protectores } \\
\text { prosocial }\end{array}$ & 0.76 \\
\hline
\end{tabular}

Nota. No se pudo realizar el análisis factorial en los FR que no tienen valores en los índices de ajuste debido al bajo número de ítems en estos dos factores (tres ítems cada uno).

\section{Puntos de corte}

Como se mencionó anteriormente, el Communities That Care Youth Survey cuenta con puntos de corte que ubican a los estudiantes en un nivel alto o bajo de riesgo y protección. Estos puntos de corte, desarrollados por el Social Development Research Group de la Universidad de Washington (Arthur, Briney, Hawkins, Abbott, Brooke-Weiss y Catalano, 2007), fueron encontrados con alta sensibilidad y especificidad en población estadounidense y minorías étnicas.

Inicialmente se utilizaron los puntos de corte de los Estados Unidos para la realización de los perfiles en las comunidades colombianas; sin embargo, en algunos casos los datos parecían contraevidentes: el riesgo disminuía con la edad y los estudiantes de grados más bajos (sexto grado) aparecían en mayor riesgo en algunos factores. Por esta razón se tomó la decisión de calcular puntos de corte para la población colombiana usando las 33.790 encuestas.

Por esta razón, se realizó el cálculo de los puntos de corte para estas comunidades, a través de la metodología de $\mathrm{Ar}$ thur et al., (2007). El procedimiento se realizó para cada FR y FP de manera individual, así como por cada grado escolar.

Con respecto a los puntos de corte de los Estados Unidos, su cálculo para las comunidades CQC mostró: (a) diferencias en los perfiles de nueve de los factores de riesgo; (b) los puntos de corte de CTC y CQC tienden a parecerse más a medida que aumenta el grado escolar en el FR comunitarios "Reglas y normas favorables al uso de drogas"; y, (c) se encontró un factor de riesgo que no se diferenciaba de manera importante, también comunitario: "Percepción so-

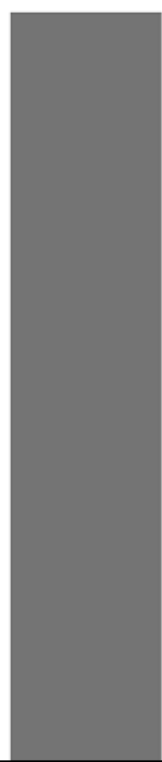

" Puntos de corte CTC
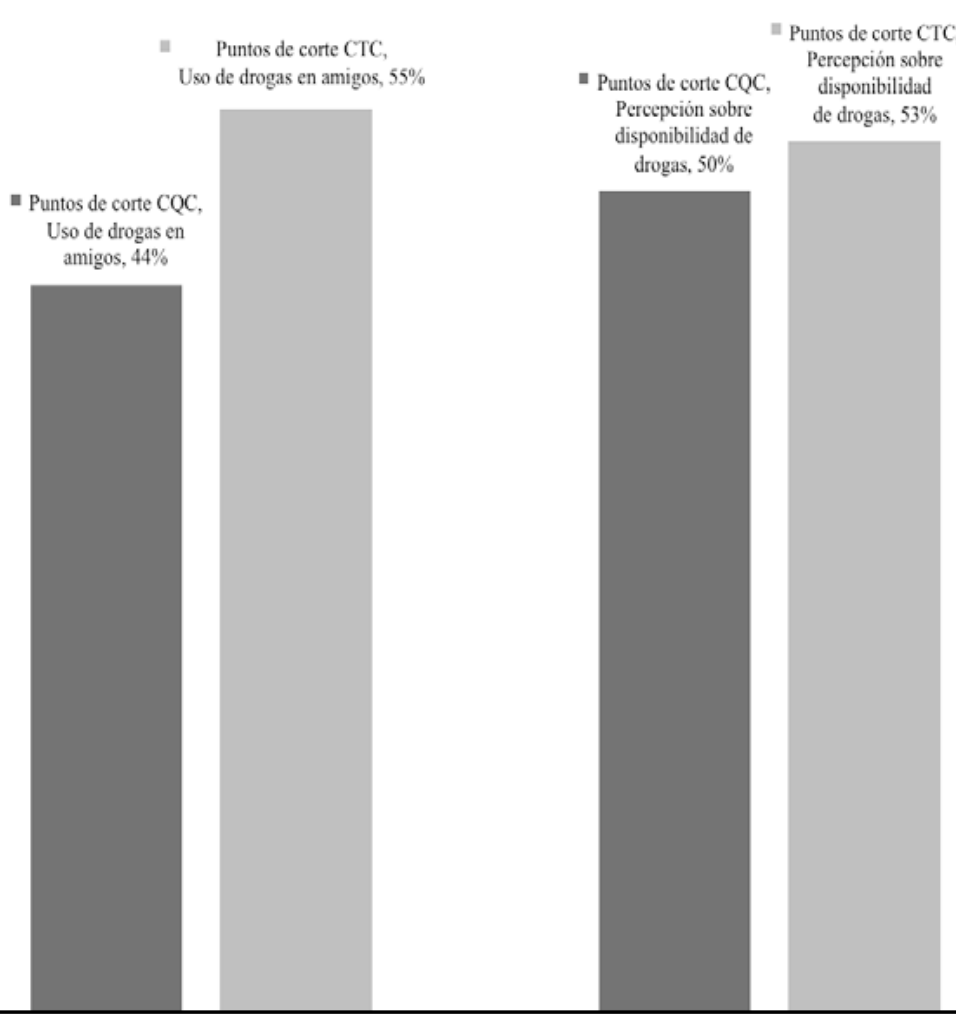

Figura 1 
Tabla 2

Comparación prevalencias de consumo de SPA de vida año y mes de los datos de la encuesta para jóvenes CQC y las prevalencias nacionales de consumo de SPA en escolares

\begin{tabular}{|c|c|c|c|c|c|c|}
\hline \multirow{3}{*}{ Sustancias Psicoactivas } & \multicolumn{6}{|c|}{ Prevalencias } \\
\hline & \multicolumn{2}{|c|}{ Vida } & \multicolumn{2}{|c|}{ Año } & \multicolumn{2}{|c|}{ Mes } \\
\hline & EJCQCa & Nacional & EJCQC & Nacional & EJCQC & Nacional \\
\hline Alcohol & $76,9 \%$ & $63,4 \%$ & $72,9 \%$ & $56,7 \%$ & $40,0 \%$ & $39,8 \%$ \\
\hline Cigarrillo & $31,0 \%$ & $24,3 \%$ & $24,9 \%$ & $16,1 \%$ & $11,3 \%$ & $9,8 \%$ \\
\hline Marihuana & $14,2 \%$ & $7,0 \%$ & $12,1 \%$ & $5,2 \%$ & $5,7 \%$ & $2,8 \%$ \\
\hline Cocaína & $1,7 \%$ & $2,4 \%$ & $1,2 \%$ & $1,9 \%$ & $0,3 \%$ & $1,0 \%$ \\
\hline Basuco & $0,6 \%$ & $0,7 \%$ & $0,4 \%$ & $0,5 \%$ & $0,1 \%$ & $0,2 \%$ \\
\hline Inhalantes & $5,1 \%$ & $3,1 \%$ & $3,5 \%$ & $1,8 \%$ & $0,9 \%$ & $0,9 \%$ \\
\hline Éxtasis & $1,4 \%$ & $1,2 \%$ & $0,9 \%$ & $0,8 \%$ & $0,1 \%$ & $0,4 \%$ \\
\hline Hongos & $2,2 \%$ & $\mathrm{~N} / \mathrm{a}$ & $1,5 \%$ & $\mathrm{~N} / \mathrm{a}$ & $0,3 \%$ & N/a \\
\hline Ácidos & $1,9 \%$ & $1,5 \%$ & $1,3 \%$ & $1,0 \%$ & $0,3 \%$ & $0,4 \%$ \\
\hline Tranquilizantes & $1,2 \%$ & $1,1 \%$ & $0,9 \%$ & $0,8 \%$ & $0,2 \%$ & $0,4 \%$ \\
\hline Popper & $1,9 \%$ & $2,5 \%$ & $1,2 \%$ & $1,4 \%$ & $0,3 \%$ & $0,6 \%$ \\
\hline Anfetaminas & $0,2 \%$ & $\mathrm{~N} / \mathrm{a}$ & $0,1 \%$ & N/a & $0,0 \%$ & N/a \\
\hline Heroína & $0,3 \%$ & $0,5 \%$ & $0,1 \%$ & $0,4 \%$ & $0,0 \%$ & $0,2 \%$ \\
\hline Dick & $5,8 \%$ & $4,1 \%$ & $3,7 \%$ & $2,7 \%$ & $0,7 \%$ & $1,3 \%$ \\
\hline
\end{tabular}

Nota. ${ }^{a}$ Encuesta para Jóvenes de Comunidades Que se Cuidan.

bre la disponibilidad de drogas". En la figura 1 se muestra un ejemplo de tres factores de riesgo de una comunidad, usando los puntos de corte preliminares calculados por la Corporación Nuevos Rumbos para CQC y los de CTC.

Es importante resaltar que estos puntos de corte se han usado solamente para presentar los datos a las comunidades CQC, debido a que estos datos están ajustados a la distribución de los datos y no corresponden a una muestra representativa del país. La Corporación Nuevos Rumbos está recolectando nuevos datos para calcular puntos de corte que puedan ser estandarizados para la población colombiana.

\section{Perfiles de riesgo y protección de las comunidades CQC}

Se recolectó información en cerca de 37.000 estudiantes de escuela secundaria, con edades entre los 11 y los 18 años, de seis municipios del Quindío y tres de Cundinamarca; los datos se obtuvieron en un total de 31 instituciones educativas, de las cuales 28 eran públicas y tres privadas. El $52 \%$ de los estudiantes era de sexo femenino y el $48 \%$ de sexo masculino. Luego de limpiar la muestra, se retuvieron 33.790 encuestas. La encuesta fue aplicada en los grados sexto a once, a la totalidad de los estudiantes que asistieron el día de la aplicación.

Como se puede observar en la Tabla 2, la población estudiada con la encuesta juvenil CQC presentó una preva- lencia de vida y año mayor que la mostrada por el estudio nacional (Ministerio de Justicia y del Derecho, Ministerio de Educación Nacional y Ministerio de Salud y Protección Social, 2011) en alcohol, cigarrillo, marihuana e inhalantes. Es importante resaltar que el consumo de marihuana en la población estudiada por CQC presenta una prevalencia de vida, año y mes que duplica los datos nacionales.

En la Tabla 3 se puede observar el perfil de riesgo y protección de los estudiantes de las comunidades estudiadas por cada grado escolar. Se encontró a nivel general que el factor de menor riesgo fue el de actitudes de los padres hacia el uso de drogas y que los FR de la comunidad parecen ser homogéneos, especialmente a medida que aumenta el grado escolar. Por otro lado, los más jóvenes parecen estar en mayor riesgo en comparación con los participantes de grados escolares superiores en los FR de pobre manejo familiar, bajo compromiso con el colegio, comportamientos antisociales en amigos y los dos FR de la comunidad.

Respecto a los factores protectores se encontró que del $40 \%$ al $50 \%$ de los participantes se encontraban en un alto nivel de protección. Otro hallazgo fue que los FP son relativamente homogéneos en todos los cursos y tienden a disminuir a medida que aumenta el grado escolar, a excepción del FP reconocimiento de la familia por involucramiento prosocial. 
Tabla 3

Perfil de riesgo y protección por grado escolar

\begin{tabular}{|c|c|c|c|c|c|c|c|}
\hline \multirow{2}{*}{ DominioCQC } & \multirow{2}{*}{ Factores de riesgo } & \multicolumn{6}{|c|}{ Porcentaje de estudiantes en riesgo por Grado escolar } \\
\hline & & 6 & 7 & 8 & 9 & 10 & 11 \\
\hline \multirow[t]{2}{*}{ Comunidad } & Percepción sobre la Disponibilidad de Drogas & $47 \%$ & $49 \%$ & $45 \%$ & $44 \%$ & $41 \%$ & $44 \%$ \\
\hline & Reglas y Normas Favorables al Uso de Drogas & $47 \%$ & $47 \%$ & $45 \%$ & $39 \%$ & $43 \%$ & $46 \%$ \\
\hline \multirow[t]{3}{*}{ Familia } & $\begin{array}{l}\text { Actitudes favorables de los padres hacia el comportamiento } \\
\text { antisocial }\end{array}$ & $42 \%$ & $36 \%$ & $43 \%$ & $42 \%$ & $40 \%$ & $40 \%$ \\
\hline & Actitudes de los padres hacia el Uso de Drogas & $31 \%$ & $39 \%$ & $31 \%$ & $36 \%$ & $40 \%$ & $44 \%$ \\
\hline & Pobre Manejo Familiar & $49 \%$ & $45 \%$ & $42 \%$ & $43 \%$ & $43 \%$ & $45 \%$ \\
\hline \multirow{7}{*}{$\begin{array}{l}\text { Pares e } \\
\text { individuo }\end{array}$} & Percepción de Riesgo del uso de Drogas de los jóvenes & $41 \%$ & $47 \%$ & $40 \%$ & $38 \%$ & $48 \%$ & $48 \%$ \\
\hline & Actitudes favorables de los jóvenes Hacia el Uso de Drogas & $39 \%$ & $41 \%$ & $37 \%$ & $45 \%$ & $49 \%$ & $37 \%$ \\
\hline & $\begin{array}{l}\text { Actitudes Favorables de los jóvenes Hacia el Comportamiento } \\
\text { Antisocial }\end{array}$ & $41 \%$ & $42 \%$ & $48 \%$ & $49 \%$ & $47 \%$ & $47 \%$ \\
\hline & Uso de Drogas en Amigos & $42 \%$ & $49 \%$ & $44 \%$ & $42 \%$ & $48 \%$ & $47 \%$ \\
\hline & Comportamientos Antisociales en Amigos & $48 \%$ & $44 \%$ & $38 \%$ & $48 \%$ & $45 \%$ & $41 \%$ \\
\hline & & \multicolumn{6}{|c|}{ Porcentaje de estudiantes en protección por Grado escolar } \\
\hline & & 6 & 7 & 8 & 9 & 10 & 11 \\
\hline \multirow[t]{2}{*}{ Familia } & Oportunidades de la familia para el involucramiento prosocial & $51 \%$ & $49 \%$ & $45 \%$ & $45 \%$ & $41 \%$ & $44 \%$ \\
\hline & Reconocimiento de la familia por involucramiento prosocial & $36 \%$ & $49 \%$ & $44 \%$ & $37 \%$ & $34 \%$ & $35 \%$ \\
\hline
\end{tabular}

\section{Discusión}

La prevención del consumo de sustancias psicoactivas ha recorrido un largo camino en sus casi 45 años de existencia. La complejidad del problema ha conducido a un número creciente de investigadores a explorar un gran número de variables y de relaciones entre ellas, generando una comprensión mucho mayor del fenómeno, de los factores que lo determinan y de las formas de influir sobre ellos a través de programas preventivos probados y efectivos (Bukowski, 2003; Hawkins et al, 2002; O’Connell, Boat y Warner, 2010; Sloboda, 2014).

CTC/CQC parece ser un sistema preventivo altamente promisorio para los países de América Latina. Aun cuando los autores (David Hawkins y Richard Catalano) siempre han considerado que la clave del éxito está en la correcta selección de los programas de prevención en la fase 5 (Haggerty y Shapiro, 2013; Haggerty, McGlynn-Wright y Klima, 2013; Hawkins et al., 2002; Shapiro, Hawkins, Oes- terle, Monahan, Brown y Arthur, 2013; Steketee, Oesterle, Jonkman, Hawkins, Haggerty y Aussems, 2013), la experiencia en Colombia muestra que las cuatro primeras fases consolidan procesos en la comunidad que ninguna otra estrategia preventiva ha logrado: solidaridad, toma de conciencia sobre la responsabilidad del futuro de los jóvenes, necesidad de identificar los factores protectores y de riesgo y de modificarlos cada vez que sea posible y necesario, y de evaluar sistemáticamente los comportamientos problemáticos y las formas de afrontarlos. Ésta será la condición necesaria para que la implementación de los planes de acción sea exitosa.

Aun cuando indiscutiblemente la fidelidad es un elemento esencial para asegurar el buen funcionamiento tanto del sistema como de los programas (Fagan, Hanson, Hawkins y Arthur, 2008; Fagan, Arthur, Hanson, Briney y Hawkins, 2011; Quinby, Hanson, Brooke-Weiss, Arthur, Hawkins y Fagan, 2008), la experiencia colombiana, al igual 
que la de algunos otros países (Jonkman, Junger-Tas y van Dijk, 2005; Jonkman, Haggerty, Steketee, Fagan, Hanson, y Hawkins, 2009; Steketee et al., 2013) muestra que hay una serie de adaptaciones que deben ser implementadas por razones de tipo cultural, de funcionamiento de las comunidades, o de condiciones y circunstancias especiales que no pueden ser previstas. En el caso de Colombia, aun cuando no se modificaron elementos que introdujeran variaciones en la estructura del sistema, sí se hicieron numerosos cambios en las rutinas de los entrenamientos, en algunos contenidos (se suprimieron y añadieron elementos según las necesidades), se modificaron tiempos (por ejemplo, se presentaron los perfiles al comienzo del proceso para despertar el interés de las comunidades) y se involucraron empleados públicos como miembros de las Juntas Comunitarias. El único punto que puede considerarse como un cambio estructural fue el inicio de la fase 5 con muy pocos programas probados y efectivos, y el facilitar que las comunidades le dieran mayor peso (transitoriamente) a las actividades que ellos mismo definían como convenientes. Nuevos Rumbos ofreció, además un conjunto de prácticas que están siendo sometidas a evaluación en el momento actual y que se basan sobre los principios que hoy se consideran como universales (NIDA, 2003; OPS, 2010).

Los resultados obtenidos mostraron que el análisis psicométrico del instrumento y de los puntos de corte es indispensable para garantizar un diagnóstico preciso. Así mismo, este proceso contribuye a la generación de tecnologías de medición en el área de la prevención en el contexto latinoamericano.

Las prevalencias de consumo de drogas como alcohol, tabaco, marihuana e inhalables, fueron muy superiores a los promedios nacionales en las poblaciones en las que se ha solicitado la implementación de CQC. El resto de sustancias tienen consumos muy similares; la excepción es la cocaína, que presenta índices de consumo menores que el promedio nacional (MJD, MEN y MSPS, 2011).

La mayoría de las comunidades tienen como factores de riego prioritario los comunitarios: la disponibilidad de drogas, y reglas y normas favorables al consumo de sustancias. En este caso un factor de confusión es la muy elevada prevalencia del consumo de alcohol y la marcada tolerancia existente en la mayoría del país con respecto a su consumo en menores; esto debe llevar a realizar análisis en los que se separen las SPA legales de las ilegales.

Hay una reflexión final que se desprende del haber estado inmersos durante más de dos años en el estudio de CTC: si bien CTC/CQC es un sistema altamente flexible, en general los programas preventivos no lo son. Tal vez la mejor prueba de ello es lo sucedido con el más famoso de todos, Life Skills Training (LST), o Habilidades para la Vida. Un estudio reciente (Luna-Adame, Carrasco-Giménez y Rueda-García, 2013), realizado con extremo rigor para evitar las críticas sobre ausencia de fidelidad, mostró resultados nulos en España en la prevención del inicio de consumo de tabaco en adolescentes, que es supuestamente el área en la que LST es más efectiva. Este dato no tiene por qué sorprender: LST fue creado hace 30 años, y no ha sufrido ninguna clase de modificaciones, mientras que el mundo ha cambiado enormemente en ese lapso, especialmente para los adolescentes; sus autores, Gilbert Botvin y Kenneth Griffin (2004), han mantenido que su programa es muy eficiente, y han rechazado las críticas, negándose a introducir cambios. Otros autores han reportado igualmente pobres resultados (Gorman, 2011; Johnson, Shamblen, Ogilvie, Collins, \& Saylor, 2009; Vicary et al., 2004, 2006). Esto mismo ocurre, probablemente, con muchos otros programas de prevención.

Sin embargo, la lógica permite pensar que esta forma de proceder condenará a todos los programas de prevención a encontrarse muy desfasados con respecto a la realidad en un plazo de pocos años, lo cual pondría en peligro toda la estructura de funcionamiento de CTC. Esto significa, en pocas palabras, que:

1. Los programas preventivos necesitan actualizarse constantemente, lo cual crea algunas dificultades a propósito de las evaluaciones;

2. Se requiere desarrollar programas preventivos que sean fácilmente modificables sin que se altere su estructura nuclear, responsable de los logros. Deberán, además, incluir procesos de evaluación flexibles y confiables.

\section{Limitaciones}

Las siguientes pueden ser consideradas las principales limitaciones de este estudio:

- Las encuestas recolectadas no constituyen una muestra representativa del país, por lo que se requerirán nuevos cálculos en otras regiones. Igualmente, es posible que requieran nuevas adaptaciones en comunidades muy diferentes, como la región Pacífica, la costa Caribe o los Llanos Orientales.

- La muy limitada disponibilidad de programas preventivos probados y efectivos pone un signo de reserva sobre los resultados que se obtendrán en la fase cinco. En la actualidad se dispone de cinco programas y hay altas probabilidades de tener en poco tiempo Miles de Manos (GIZ) y Familias Unidas (Universidad de Miami).

- No hay datos de archivo confiables fuera de las ciudades grandes.

Este trabajo debe considerarse una invitación a ensayar en América Latina y en el Caribe uno de los mejores sistemas de prevención existentes. Además de todas las ventajas mencionadas hasta ahora, los costos de implementación son modestos y posee su propia estrategia de evaluación, lo cual facilitará la realización de los ajustes y permitirá hacer comparaciones e identificar con precisión lo que pueda ser modificable y lo que no. 


\section{Conflictos de interés}

No se tienen conflictos de interés.

\section{Referencias}

Aja-Eslava, L. y Gómez-Avila, J. J. (2013). Evaluación y seguimiento de un programa de prevención de consumo de SPA y riesgo de suicidio: 1999-2012. Típica: Boletín electrónico de Salud Escolar, 9, 31-42.

Arthur, M., Hawkins, D., Pollard, J., Catalano, R. y Baglioni A. (2002). Measuring risk and protective factors for substance use, delinquency and other adolescent problems behaviors: The Communities That Care Youth Survey. Evaluation Review, 24, 575-601. doi: 10.1177/019384102237850.

Arthur, M., Briney, J., Hawkins, D., Abbot, R., Brooke-Weiss, B. y Catalano, R. (2007). Measuring risk and protection in communities using the Communities That Care Youth Survey. Evaluation and Program Planning, 30, 197-211. doi: 10.1016/je.evalprogplan.2007.01.009

Arthur, M.W., Hawkins, J.D., Catalano, R.F. y Olson, J.J. (2002). Community Key Informant Survey. Seattle: Social Development Research Group, University of Washington

Arthur, M. W., Hawkins, J. D., Brown, E. C., Briney, J. S., Oesterle, S. y Abbott'R. D. (2010). Implementation of the Communities That Care prevention system by coalitions in the Community Youth Development Study. Journal of Community, 38, 245-258.

Byrne, B. (2012). Structural equational modeling with Mplus: basic concepts, applications, and programming. New York: Routledge.

Botvin, G. J. y Griffin, K.W. (2004). Life Skills Training: empirical findings and future directions. The Journal of Primary Prevention, 25, 211-232.

Briney, J., Brown, E., Hawkins, D. y Arthur, M. (2012). Predictive validity of established cut points for risk and protective factor scales form the Communites That Care Youth Survey. Journal of Primary Prevention, 33, 249-258. doi: 10.1007/s10935-012-0280-1

Brooke-Weiss, B., Haggerty, J. P., Fagan, A. A., Hawkins, J. D. y Cady, R. (2008). Creating Community Change to Improve Youth Development: The Communities That Care System. The Prevention Researcher, 15, 21-24.

Brown, E. C., Hawkins, J. D., Arthur, M. W., Briney, J. S. y Abbott, R. D. (2007). Effects of Communities That Care on Prevention Services Systems: Findings From the Community Youth Development Study at 1.5 Years. Prevention Science, 8, 180-191.

Brown, E. C., Graham, J. W., Hawkins, D. J., Arthur, M. W., Baldwin, M. M., Oesterle, M., Briney, J. S., Catalano, R. F. y Abott, R. D. (2009). Design and Analysis of the Community Youth Development Study Longitudinal Cohort Sample. Evaluation Review, 33, 311-334.
Brown, E. C., Hawkins, J. D., Arthur, M. W., Briney, J. S. y Fagan, A. A. (2011). Prevention service system transformation using Communities That Care. Journal of Community Psychology, 39, 183-201.

Bukoski, W. J. (2003). The emerging science of drug abuse prevention. En Z. Sloboda y W.J. Bukoski (Eds.).Handbbook of Drug Abuse Prevention: theory, science and practice (pp.3-26). New York: Klewer Academic/Plenum Publishers.

Catalano, R. F., Arthur, M. W., Hawkins, J. D., Berglund, L. y Olson, J. J. (1998). Comprehensive community and school based interventions to prevent antisocial behavior. En R. Loeber y D.P. Farrington (eds), Serious and violent juvenile offenders: risk factors and succesfull interventions (pp. 248-284). Thousand Oaks, CA: Sage.

Fagan, A. A., Hanson, K., Hawkins, J. D. y Arthur, M. W. (2008). Bridging Science to Practice: Achieving Prevention Program Implementation Fidelity in the Community Youth Development Study. American Journal of Community Psychology, 41, 235-249.

Fagan, A. A., Hanson, K., Hawkins, J. D. y Arthur, M. W. (2008). Implementing Effective Community-Based Prevention Programs in the Community Youth Development Study. Youth Violence and Juvenile Justice, 6, 256-278.

Fagan, A. A, Arthur, M. W, Hanson, K., Briney, J. S. y Hawkins, J. D. (2011). Effects of Communities That Care on the adoption and implementation fidelity of evidence-based prevention programs in communities: results from a randomized controlled trial. Prevention Science, 12, 223-234.

Fagan, A. A., Van Horn, M. L., Hawkins, J. D. y Arthur, M., (2007). Using community and family risk and protective factors for community-based prevention planning. Journal of Community Psychology, 35, 535-555.

Feinberg, M. E., Greenberg, M. T., Osgood, D. W., Sartorius, J. y Bontempo, D. (2007). Effects of the Communities That Care model in Pennsylvania on youth risk and problem behaviors. Prevention Science, 8, 261-270.

France, A. y Crow, I. (2005). Using the 'risk factor paradigm' in prevention: lessons from the evaluation of Communities that Care. Children y Society, 19, 172-184.

Glaser, R. R., Van Horn, L., Arthur, M. W., Hawkins, J. D. y Catalano, R. F. (2005). Measurement Properties of the Communities That Care Youth Survey Across Demographic Groups. Journal of Quantitative Criminology, 21, 73102. doi: 10.1007/s10940-004-1788-1

Gorman, D. (2011). Does the Life Skills Training program reduce use of marijuana? Addiction Research $\mathcal{E}^{2}$ Theory, 19, 470-481.

Haggerty, K. P. y Shapiro, V. B. (2013). Science-based prevention trough Communities That Care: a model of social work practie for public health. Social Work in Public Health. Special Issue: The role of Social Work in the Prevention and Treatment of Substance Use Dirsorders, 28, 349-365. 
Haggerty, K. P., McGlynn-Wright, A. y Klima, T. (2013). Promising parenting programs for reducing adolescent problem behaviors. Journal of Children's Services, 8, 229-243.

Harris, S., Csémy, L., Sherritt, L., Starostova, O., Van Hook, S., Johnson, J., ... y Knight, J. (2012). Computer-Facilitated Substance Use Screening and Brief advice for teens in primary care: An international trial. Pediatrics, 129, 1072-1082.

Hawkins, J. D. (2006). Science, Social Work, Prevention: Finding the Intersections. Socisal Work Research, 30, 137152.

Hawkins, J. D., Catalano, R. y Arthur, M. W. (2002). Promoting science-based prevention in communities. Addictive Behaviors, 27, 951-976.

Heene, M., Hilbert, S., Draxler, C., Ziegler, M. y Bühner, M. (2001). Masking misfit in confirmatory factor analysis by increasing unique variances: a cautionary note on the usefulness of cutoff values of fit indices. Psychological Methods, 16, 319- 336. doi: 10.1037/a0024917

Hu, L. T. y Bentler, P. M. (1999). Cutoff criteria for fit indexes in covariance structure analysis: conventional criteria versus new alternatives. Structural Equation Modeling. 6, 1-55.

Jonkman, H. B., Junger-Tas, J. y van Dijk, B. (2005) From Behind Dikes and Dunes:Communities that Care in theNetherlands. Children E Society, 19, 105-116.

Jonkman, H. B., Haggerty, K. P., Steketee, M., Fagan, A. A., Hanson, K. y Hawkins, J.D. (2009). Communities That Care, Core Elements and Context: Research of Implementation in Two Countries. Social Development Issues, $30,42-57$.

Johnson, K. W., Shamblen, S. R., Ogilvie, K. A., Collins, D. y Saylor, B. (2009). Preventing youths' use of inhalants and other harmful legal products in frontier Alaskan communities: A randomized trial. Prevention Science, 10, 298-312.

Knight, J. R., Sherritt, L., Harrys, S. K., Gates, E. C. y Chang, G. (2003). Validity of Brief alcohol screening tests among adolescents: A comparison of the AUDIT, POSIT, CAGE, and CRAFFT. Alcoholism: Clinical and Experimental Research, 27, 67-73.

Luna-Adame, M., Carrasco-Giménez, T. J. y Rueda-García, M. M. (2013). Evaluation of the effectiveness of a smoking prevention program based on the 'Life Skills Training' approach. Health Education Research, 28, 673-682.

Ministerio de Justicia y del Derecho., Ministerio de Educación Nacional, y Ministerio de Salud y Protección Social (2011). Estudio Nacional de Consumo de Sustancias Psicoactivas en Población Escolar. Recuperado de http://www. unodc.org/documents/colombia/Documentostecnicos/Estudio_Consumo_Escolares.pdf.

Monteiro, M. G. (2013). Alcohol y salud pública en America Latina: ¿cómo impedir un desastre sanitario?. Adicciones, 25, 99-105.
Nationl Institute on Drugs Abuse. (2003). Preventing drug use among children and adolescents. Recuperado de http:/ / www.drugabuse.gov/sites/default /files/preventing druguse_2.pdf

O'Connell, M. E., Boat, T. y Warner, K. E. (2010). Preventing mental, emotional and behavioral disorders among young people. Washington, D.C.: The National Academies Press.

Organización Panamericana de la Salud. (2010). La política de drogas y el bien público. Recuperado de http:// www.cicad.oas.org/fortalecimientoinstitucional/lanesnacionales /docs/ La\%20politica\%20de\%20drogas $\% 20 \mathrm{y} \% 20 \mathrm{el} \% 20 \mathrm{bien} \% 20$ publico.pdf

Pérez-Gómez, A. y Scoppetta Díaz-Granados, O. (2011) El CRAFFT/CARLOS como Instrumento para la Identificación Temprana de Consumo de Alcohol y Otras SPA: una Adaptación al Español. Revista Colombiana de Psicología, 20, 265-274.

Pérez Gómez, A. (2013). Columbia: fuera del ojo del huracán, pero todavía cerca. Adicciones, 25, 106-108.

Quinby, R. K., Hanson,K., Brooke-Weiss, B. L., Arthur, M. W., Hawkins, J. D. y Fagan, A. A.(2008). Installing the Communities That Care prevention system:I mplementation progress and fidelity in a randomized controlled trial. Journal of Community Psychology, 36, 313-332.

Rhew, I. C., Brown, E., Hawkins, D. y Briney, J. (2013). Sustained effect of the Communities That care system on prevention service system transformation. American Journal of Public Health, 103, 529-535.

Ringwalt, C., Vincus, A. A., Hanley, S., Ennett, S. T., Bowling, J. M. y Haws, S. (2011). The Prevalence of Evidence-based Drug Use Prevention Curricula in U.S. Middle Schools in 2008. Prevention Science, 12, 63-69

Shapiro, V. B., Hawkins, J. D., Oesterle, S., Monahan, K. C., Brown, E. C. y Arthur, M. W. (2013). Variation in the effect of Communities That Care on community adoption of a scientific approach to prevention. Journal of the Society for Social Work and Research, 4, 154-164.

Social Development Research Group. (2014). Social Development Research Group. Recuperado de http://www. sdrg.org.

Sloboda, Z. (2014). Reconceptualizando los procesos de prevención del consumo de drogas. Adicciones, 26, 3-9.

Steketee, M., Oesterle, S., Jonkman, H., Hawkins, J. D., Haggerty, K. P. y Aussems, C. (2013). Transforming prevention systems in the United States and the Netherlands using Communities That Care Promising prevention in the eyes of Josine Junger-Tas. European Journal of Criminal Policy and Research, 19, 99-116.

Substance Abuse and Mental Health Services Administration. (1999). Brief Interventions and Brief Therapies for Substance Abuse. Treatment Improvement Protocol (TIP) Series, No. 34. Rockville (MD): Substance Abuse and Mental Health Services Administration (US). 
Vicary, J. R., Henry, K. L., Bechtel, L. J., Swisher, J. D., Smith, E. A., Wylie, R. y Hopkins, A. M. (2004). Life skills training effects for high and low risk rural junior high school females. Journal of Primary Prevention, 25, 399-416.

Vicary, J. R., Smith, E. A., Swisher, J. D., Hopkins, A. M., Elek, E., Bechtel, L. J. y Henry, K. L. (2006). Results of a 3-year study of two methods of delivery of life skills training. Health Education E Behavior, 33, 325-339. 